\title{
Preparation of ultrafine iron phosphate micro-powder from phosphate slag
}

\author{
Fangjing Sun ${ }^{1,2}$, Yi Zhang1 ${ }^{1}$ Jiawei Zhang ${ }^{1}$, Xixi Yan ${ }^{1}$, Xiaoyu Liu'1,2, Lijun Wang1,2,a \\ ${ }^{1}$ School of Environmental and Materials Engineering, Shanghai Polytechnic University, Shanghai 201209, P. R. China; \\ ${ }^{2}$ Research Center of Resource Recycling Science and Engineering, Shanghai Polytechnic University, Shanghai 200444, China.
}

\begin{abstract}
In this experiment, ultrafine iron phosphate micro-powder was prepared by hydrothermal method which used phosphate slag as an iron source. The effects of reaction temperature, surfactants type and amount on its particle size were explored. The samples were characterized by using Malvern Laser Particle Size Analyzer (MS2000), X-Ray Diffractometer (XRD), Scanning Electron Microscope (SEM) and Energy Dispersive X-Ray Spectroscopy (EDX). The results showed that at $160{ }^{\circ} \mathrm{C}, 1 \mathrm{wt} \% \mathrm{CTAB}$, monoclinic iron phosphate micro-powder was obtained with an average particle size about $0.4 \mu \mathrm{m}$ which also has a good dispersion in aqueous solution.
\end{abstract}

\section{Introduction}

With the improvement of people's living standards, transportation such as cars, ships and airplanes have gradually entered our lives. And the requirements for people incorrosion resistance and service life of their metal parts have become highest than ever before ${ }^{[1]}$. In order to prevent the metal surface being corroded and improve its wear resistance, phosphating technology has been widely used in the steel industry ${ }^{[2]}$. Phosphorization is one of the key processes in the metal industry before painting ${ }^{[3-5]}$. Its function is to improve the adhesion and wear resistance of the coating effectively. However, along with the phosphating process, the production of phosphate slag will be inevitable $e^{[6-8]}$. The production of phosphate slag not only reduces the quality of the workpiece, but also affects the phosphating process, and if it is directly discharged, it will cause serious pollution to the soil, water and air ${ }^{[9-13]}$. More importantly, the phosphating slag contains a large amount of $\mathrm{Fe}^{3+}, \mathrm{Zn}^{2+}$, $\mathrm{PO}_{4}{ }^{3-}$, etc., which are all recyclable materials ${ }^{[14-16]}$. On the one hand it can be used to reconfigure the phosphating solution, on the other hand, it can be used to prepare iron phosphate $^{[17-19]}$. In recent years, China's environmental problems have aroused great concerns from the government. The people's awareness of environmental protection has greatly deepened. The treatment and disposal of phosphate slag has become one of the hotspots of research in recent years.

At present, some researches have been done on the utilization of phosphate slag both internally and externally ${ }^{[17-18]}$. The commonly used methods are: physical method (adsorption, reverse osmosis, electrodialysis and contact filtration method), chemical precipitation method, biological method, etc ${ }^{[19-24]}$. However, based on the treatment technology of phosphate slag is not perfect, many companies still use simple accumulation, incineration or landfill method ${ }^{[2]}$. This subject has studied the composition and properties of phosphate slag and the synthetic process of recycling and reuse. It has a deep understanding of it and has mastered the technology of phosphate slag to purify iron phosphate ${ }^{[5,8,25-26]}$. I have adopted a new method to purify and prepare iron phosphate micro-powder with smaller particle size. The purified and modified iron phosphate micro-powder has high dispersibility and can be used as catalyst $^{[15,25]}$, adsorbent and slow release fertilizer ${ }^{[26]}$. It can also be used as a precursor material for the preparation of lithium iron phosphate cathode ${ }^{[27]}$. Through the purification and utilization of iron phosphate in the phosphate slag, thousands of tons of iron phosphate can be recovered every year, and the economic value created can reach millions, at the same time it also solves a major problem of environmental pollution.

In summary, the subsequent research and application of the purified iron phosphate has theoretical research value and practical application value for promoting the phosphate slag resource treatment.

\section{Materials and Methods}

\subsection{Experimental equipment and Chemicals}

\subsubsection{Experimental equipment}

a Corresponding author: ljwang@ @spu.edu.cn 
Experimental Equipment: GSH type reaction kettle (Taixing City Construction Company); HJ-6 multi-head magnetic stirrer; SHB-III circulating water multi-purpose vacuum pump and DNF-6021 vacuum drying oven.

Characterization and performance test Equipment: XRay Diffractometer Diffractometer (XRD) (D8-Advance, Bruker Corporation, Germany) $(\lambda=0.154056 \mathrm{~nm})$; Scanning Electron Microscope (SEM) (S-4800, Hitachi Corporation, Japan); Malvern 2000 Laser Particle Size Instrument (MS2000); American Micromeritics ASAP2020M Specific Surface Analyzer (BET); Japan Shimadzu 720 X-ray fluorescence spectrometer (EDX).

\subsubsection{Chemicals}

phosphate slag (Zhejiang Haiyan Metal Products Co., Ltd.); phosphoric acid (analytical grade, content $\geq 85.0$ wt\%); Cationic surfactant (CTAB), Analytically pure; Anionic surfactant (SDS), Analytically pure; Amphoteric surface activity Agent (hexamethylenetetramine), Analytically pure; Anhydrous ethanol, Analytically pure and Deionized water.

\subsection{Experimental method}

(1) Weigh a certain amount of purified phosphating slag in a beaker, add concentrated phosphoric acid (85.0 wt\%), cationic surfactant $(\mathrm{CTAB})$ and deionized water in a certain proportion and stir evenly under a magnetic stirrer; (2) Re-transfer the reaction vessel, hydrothermal reaction for $12 \mathrm{~h}$, vacuum filtration, drying at $80{ }^{\circ} \mathrm{C}$ to obtain ultra-fine iron phosphate $\left(\mathrm{FePO}_{4} 2 \mathrm{H}_{2} \mathrm{O}\right)$;

(3) Calcined at $250{ }^{\circ} \mathrm{C}$ for $6 \mathrm{~h}$ to obtain a pale yellow anhydrous iron phosphate sample.

It was found by the Malvern 2000 laser particle size analyzer that the obtained powder sample had a particle size of about $0.4 \mu \mathrm{m}$.

\section{Results and discussion}

\subsection{Effect of reaction temperature on the particle size of iron phosphate micropowder}

The cationic surfactant (CTAB) was selected as the dispersant. The effect of hydrothermal temperature on the particle size of iron phosphate was studied under the condition of controlling the CTAB amount to $0.4 \mathrm{~g}$ and the feed ratio being 4:2:1:1. It can be seen from Fig. 1 that the particle size of iron phosphate decreases first and then increases when the temperature rises. At $160{ }^{\circ} \mathrm{C}$, the particle size is the smallest, which the average particle size is about $0.4 \mu \mathrm{m}$. The reason for this trend may be: before $160{ }^{\circ} \mathrm{C}$, as the temperature increases, the nucleation rate of the crystal grains also increases, and the crystal nucleus is too late to grow. At this time the particle size is getting smaller and smaller, along with the temperature increasingly, the number of crystal nuclei increases sharply, the molecular motion is severe.
However, the probability of collision between crystal grains and crystal grains increases, so that agglomeration occurs, and the particle size of the iron phosphate particles becomes larger.

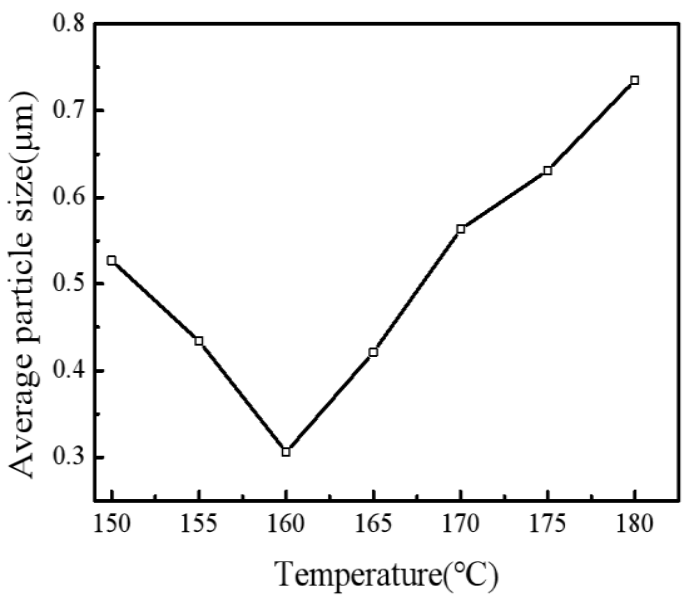

Figure 1. Effect of reaction temperature on particle size of iron phosphate micro-powder

\subsection{Effect of reaction ratio on the particle size of iron phosphate}

During the whole reaction process, the reaction ratio of purified iron phosphate and phosphoric acid, absolute ethanol and deionized water also has a great influence on the particle size. The reaction temperature is controlled at $160^{\circ} \mathrm{C}$ for $12 \mathrm{~h}$. Under this condition, the influence of the feed ratio of the raw materials on the particle size was explored. It can be seen from Table 1 that the particles of the particles were purified when the iron $\mathrm{FePO}_{4}$ : $\mathrm{H}_{3} \mathrm{PO}_{4}: \mathrm{C}_{2} \mathrm{H}_{6} \mathrm{O}: \mathrm{H}_{2} \mathrm{O}=4: 2: 1: 1$ was purified and the particle size is the smallest. At the same time, it can be seen that the content of phosphoric acid and deionized water have significant influences on the particle size. When no water is added, the particle size is large, this probably because the deionized water provides a liquid environment for its nucleation and together with it achieved a dispersion effect.

Table 1 Effect of reaction ratio on the particle size of iron phosphate

\begin{tabular}{|c|c|c|c|c|c|}
\hline & \multicolumn{2}{|c|}{$\mathrm{FePO}_{4} \cdot 2 \mathrm{H}_{2} \mathrm{O}$} & $\mathrm{H}_{3} \mathrm{PO}_{4}$ : & $\mathrm{C}_{2} \mathrm{H}_{6} \mathrm{O}$ : & $\mathrm{H}_{2} \mathrm{O}$ \\
\hline $\begin{array}{c}\text { Reaction } \\
\text { ratio }\end{array}$ & $4: 1: 2: 0$ & $4: 1: 2: 1$ & $4: 2: 1: 0$ & $4: 2: 1: 1$ & $4: 2: 2: 0$ \\
\hline $\begin{array}{c}\text { Average } \\
\text { particle } \\
\text { size } \\
(\mu \mathrm{m})\end{array}$ & 0.705 & 0.697 & 0.581 & 0.407 & 0.671 \\
\hline
\end{tabular}

\subsection{Effect of the types of surfactant on the particle size of iron phosphate micropowder}

Surfactants are mainly classified into three major categories: cationic surfactant, anionic surfactant, and zwitterionic surfactant. This process controls the 
hydrothermal reaction temperature at $160{ }^{\circ} \mathrm{C}$ for $12 \mathrm{~h}$, and three types of surfactant is used as dispersant to regulate the particle size of iron phosphate. The most commonly used surfactant is: Cetyltrimethyl Ammonium Bromide (CTAB), Sodium Dodecyl Sulfate (SDS) and Dodecyl Sulfopropyl Betaine (DSB). As shown in Figure 2 below, the three surfactants have different effects on the particle size of iron phosphate under different surfactant dosages. The results show that the cationic surfactant can effectively control the growth of the nucleation of the iron phosphate micropowder and can disperse the fine powder of iron phosphate well, while the anion and zwitterion have a weaker regulation effect on the iron phosphate particle size. The reason may be that the phosphate anion moiety binds to the cationic surfactant to prevent the growth of the iron phosphate particles.

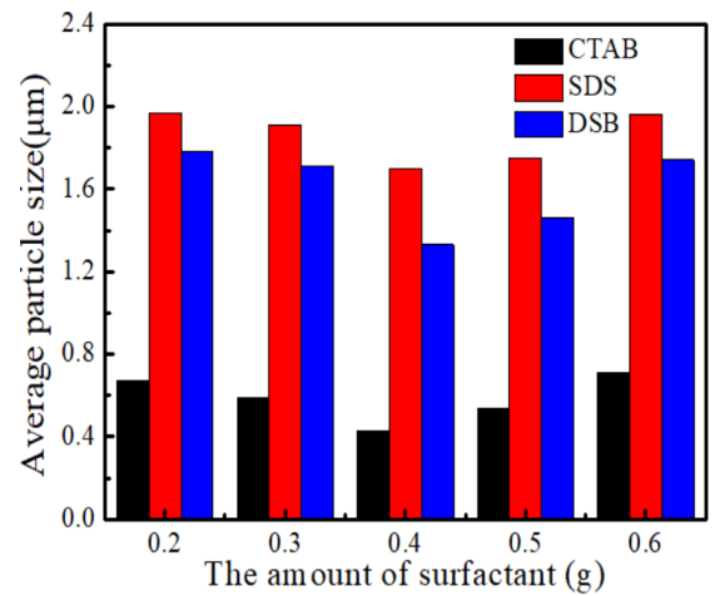

Figure 2. Effect of the types of surfactant on the particle size of iron phosphate micro-powder

\subsection{Effect of the amount of cationic surfactant CTAB on the particle size of iron phosphate micropowder}

Through the above comparison, we have used cationic surfactant (CTAB) as the preferred surfactant, and the amount of surfactant is one of the factors affecting the particle size. When other reaction conditions are not changed, the influence of the amount of CTAB on its particle size can be investigated. As shown in Fig.2, it can be visually seen that as the amount of CTAB increases, the particle size of the obtained sample first decreases and then increases. When the amount of CTAB is $0.4 \mathrm{~g}$, that is $1 \mathrm{wt} \%$ of the amount of iron phosphate, the particle size of the sample is minimized. The reason why the particle size is first reduced and then increased may be that when the amount of CTAB is small, a thin film can be formed on the surface of the sample to hinder the formation of other chemical bonds, and the growth of crystal grains is limited, and the particle size is very small. When the amount of CTAB is too large, the surfactant coated on the surface of the sample accumulates, and the van der Waals force between the CTAB molecules causes them to stick together, causing the entire particle to become larger.

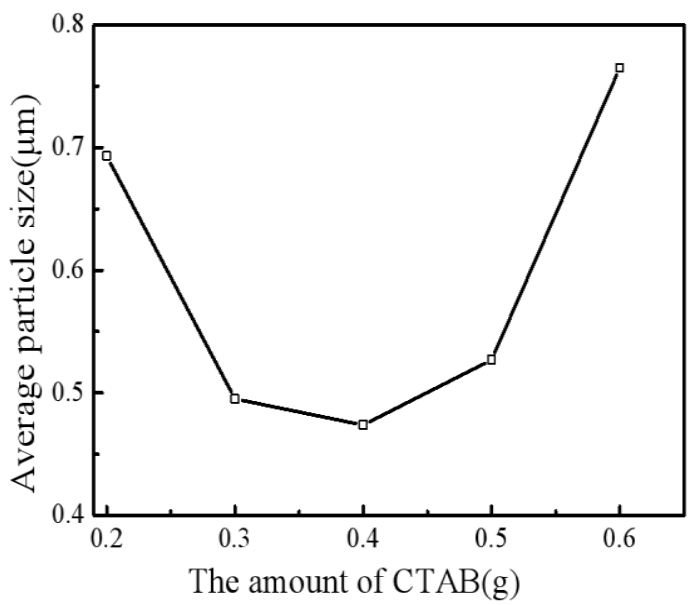

Figure 3. Effect of CTAB amount on particle size of iron phosphate micro-powder

\subsection{Characterization}

\subsubsection{SEM of sample}

According to the above experimental investigation, the optimal purification conditions for the preparation of iron phosphate micropowder were selected. As shown in Fig. 3 , the hydrothermal temperature was $160{ }^{\circ} \mathrm{C}$, and the reaction ratio was: after the purification, iron phosphate: phosphoric acid: ethanol: water $=4: 2: 1: 1$, the reaction time is $12 \mathrm{~h}$, the SEM image of the sample prepared under the condition that the amount of surfactant (CTAB) is $1 \mathrm{wt} \%$ It can be seen that the particle size of the sample is relatively uniform, relatively scattered. The average particle size is about $0.4 \mu \mathrm{m}$.

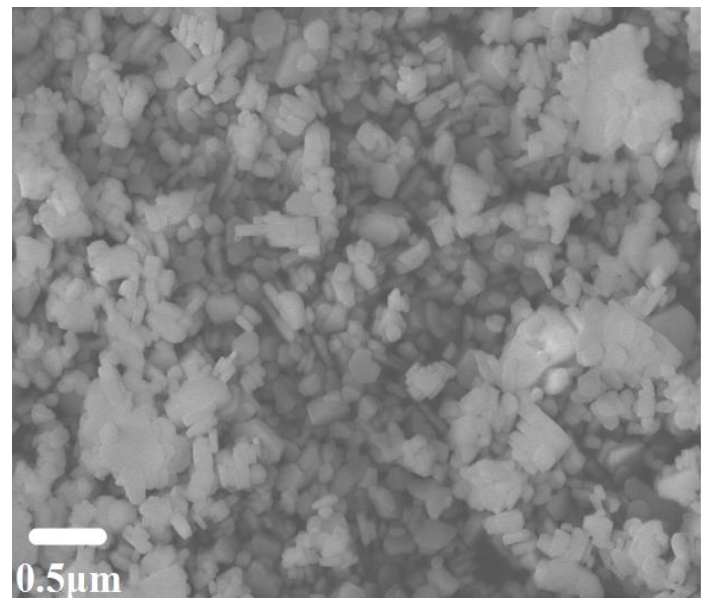

Figure 4. Hydrothermally prepared sample SEM image

\subsubsection{XRD of sample}

Fig.5 is a comparison of the hydrothermally prepared sample and the standard iron phosphate XRD. The prepared sample spectrum is consistent with the standard spectrum, and the peaks of the three strong peaks are sharp The XRD test results indicates that the micropowder belongs to Monoclinic crystal compared to the standard PDF card 33-0666. 


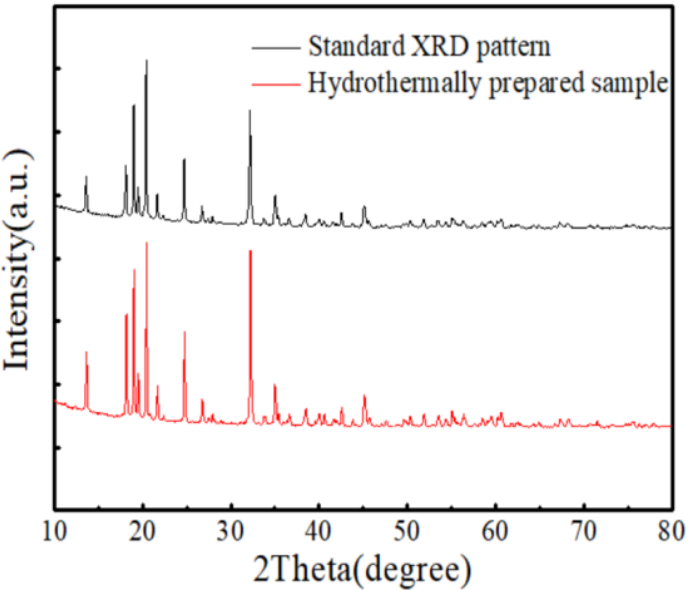

Figure 5. Hydrothermally prepared samples and iron phosphate standard XRD spectra

\section{Summary}

In this paper, the solid waste phosphating slag is purified by a simple process, in order to prepare micron-sized iron phosphate powder. The XRD test results show that the prepared iron phosphate belongs to the monoclinic system, which is a regular-sized, flat-surfaced micronsized sheet with a thickness. The thickness thereof is about $200 \mathrm{~nm}$, and the average particle diameter is about $0.4 \mu \mathrm{m}$.

In this experiment, the particle size is controlled by changing the reaction temperature, the type and amount of surfactant, and after a large number of experiments, relatively preferred conditions are obtained: purified iron phosphate: phosphoric acid: absolute ethanol: deionized water $=4: 2: 1: 1$, the hydrothermal temperature is $160{ }^{\circ} \mathrm{C}$, the reaction time is $12 \mathrm{~h}$, and the amount of the surfactant $(\mathrm{CTAB})$ is $1 \mathrm{wt} \%$ of the amount of iron phosphate.

This experiment provides new ideas and new methods for the resource treatment of phosphating slag. The prepared iron phosphate has a good application in the fields of agriculture, ceramics, environmentally friendly coatings and surface passivation.Because of its unique adsorption performance, ion exchange performance and electrochemical performance, it has also become an increasingly important application in the fields of industrial wastewater and lithium battery electrode materials.

\section{Acknowledgments}

This work was supported by Engineering Key Subject of Shanghai Polytechnic University (No. XXKZD1601), Shanghai Polytechnic University Graduate Program Foundation (EGD16YJS012) and Gaoyuan Discipline of Shanghai-Environmental Science and Engineering (Resource Recycling Science and Engineering). The authors acknowledge the use of equipment, and the scientific and technical assistance of Research Center of Resource Recycling Science and Engineering, Shanghai Polytechnic University.

\section{References}

[1] X Y Wu, J C Huang, Q C Lang, et al. Progress and prospects of research on phosphating slag resource[J].Recy. Circ.8(8): 38-41(2015).

[2] X Y Xiong, T Q Xiong, Q Q Song, et al. Metal surface treatment phosphati slag comprehensive utilization technology[J]. Meta. Pow. 2: 43-49(2015).

[3] Y Lu, Y T Song, Y L Liu. Study on preparation and properties of phosphate corrosion resistant coatings $[\mathrm{J}]$. Elect. Rev, 30(118): 19-22.(2016).

[4] H Q Fan, S Y Li. Research progress on the status and resource utilization of solid waste phosphate $\operatorname{slag}[\mathrm{J}]$. Mode. Chem. 27(s1): 97-99, 101(2007).

[5] X Q Liu, S N Zhang, L J Wang, et al. Effect of calcination temperature on preparation of $\mathrm{LiFePO} 4 / \mathrm{C}$ positive electrode material by purifying phosphate Slag $[\mathrm{J}]$. Journal S. P. U.34(1): 26-30(2017).

[6] F P Ma, X W Chen, J Tian. Production technology of tricalcium phosphate by using phosphating waste residue[J]. Inor S. I. 47(9): 54-55(2015).

[7] J T, X W Chen, R P Jiang. Zinc phosphating solution waste slag comprehensive utilization technology[J]. Mode C. I. 35(6): 155-156(2015).

[8] D F Wu, S N Zhang, L J Wang, et al.Preparation of new catalyst and photocatalytic reaction for waste phosphate slag hydrothermal reaction[J]. Journal S. P. U.33(4): 283-288(2016).

[9] H Y Ye, D M Zheng, H Q Li, et a.Study on preparation of ultrafine dihydrate ferric phosphate[J].Inor S. I. 44(4): 59-61(2012).

[10] P Z Zhao, H B Liu, H H Zheng, et al. Facile synthesis of $\mathrm{FePO}_{4} \cdot 2 \mathrm{H}_{2} \mathrm{O}$ submicrometer-discs. Mate L. 123:128-130(2014).

[11] H J Song, Y L Sun, X H Jia. Hydrothermal synthesis of iron phosphate microspheres constructed by mesoporous polyhedral nanocrystals. Mater C. 107: 182188(2015).

[12] G C Li, S Zhang, H G Peng, et al. Growth and shape control of orthorhombic $\mathrm{Fe}_{5}\left(\mathrm{PO}_{4}\right)_{4}(\mathrm{OH})_{3} \cdot 2 \mathrm{H}_{2} \mathrm{O}$ single crystalline dendrites. Jour C. G. 312(20): $2967-$ 2971(2010).

[13] Y M Zhu, S Z Tang, H H Shi, et al . Synthesis of $\mathrm{FePO}_{4} \bullet \mathrm{xH}_{2} \mathrm{O}$ for fabricating submicrometer structured $\mathrm{LiFePO}_{4} / \mathrm{C}$ by a co-precipitation method. Cerams Interl, 40(2): 2685-2690(2014).

[14] T B Zhang, X B Cheng, Q Zhang, et al. Construction of a cathode using amorphous $\mathrm{FePO}_{4}$ nanoparticles for a high-power/energy-density lithiumion battery with long-term stability. Jour P. S. 324: 5260(2016)

[15] F K Behbahani, F MGolchin. A new catalyst for the synthesis of 2-substituted perimidines catalyzed by $\mathrm{FePO}_{4}$. Jour T.U. Sci. 11(1): 85-89(2017).

[16] X Wang, J Zhuang, Q Peng, et al. A general strategy for nanocrystal synthesis, Nature,437: 121-124 (2005).

[17] L S Zhong, J S Hu, H P Liang, et al. Selfassembled 3D flowerlike iron oxide nanostructures and their application in water treatment, Adv. Mater. 18: 2426-2431(2006). 
[18] C Chen, W Chen, J Lu, et al.Transition-metal phosphate colloidal spheres, Angew. Chem. Int. Ed. 48: 4816-4819(2009).

[19] H Okawa, J P Yabuki, Y H Kawamura, et al. Synthesis of $\mathrm{FePO}_{4}$ cathode material for lithium ion batteries by a sonochemical method, Mater. Res. Bull. 43: 1203-1208(2008).

[20] A Mamoru. Oxidation activity of iron phosphate and its characters, Catal. Today 85: 193-198(2003) .

[21] K Kandori, T Kuwae, T Ishikawa. Control on size and adsorptive properties of spherical ferric phosphate particles, J. Colloid Interface Sci. 300: 225-231 (2006).

[22] Z C Shi, A Attia, W L Ye, et al. Synthesis of $\mathrm{FePO}_{4}$ by direct solid state reaction at ambient temperature and its charge-discharge properties, Electrochim. Acta 53: 2665-2673 (2008).

[23] F Cao, D X Li. Biotemplate synthesis of monodispersed iron phosphate hollow microspheres, Bioinsp. Biomim. 5: 16005-16010 (2010)

[24] L M Wei, Y Zhang, L J Wang, et al. Synthesis of nitrogen-doped carbon

nanotubes- $-\mathrm{FeO}_{4}$ composite from phosphate residue and its application as effective Fenton-like catalyst for dye degradation. Jour E. Sci,1-9(2018).

[25] Y Zhang, Z F Ying, L J Wang, et al. Modified iron phosphate/polyvinyl alcohol composite film for controlled-release fertilisers[J]. RSC Adv.8: 1814618152(2018).

[26] R Cai, Y P Du, W Y Zhang, et al. Synthesis of porous amorphous $\mathrm{FePO}_{4}$ nanotubes and their lithium storage properties, Chem. Eur. J. 19:1568-1572(2013) . 